\title{
Echocardiographic Screening Detects a Significant Burden of Rheumatic Heart Disease in Australian Torres Strait Islander Children and Missed Opportunities for its Prevention
}

\author{
Allison Hempenstall, ${ }^{1,2}$ Erin Howell, ${ }^{3}$ Katherine Kang, ${ }^{3}$ Ken W. T. Chau, ${ }^{3}$ Amylou Browne,,${ }^{1}$ Ella Kris, ${ }^{1,2}$ Hylda Wapau, ${ }^{1}$ Pelista Pilot, ${ }^{1}$ \\ Simon Smith, ${ }^{3}$ Benjamin Reeves, ${ }^{3}$ and Josh Hanson ${ }^{3,4 *}$ \\ ${ }^{1}$ Torres and Cape Hospital and Health Service, Thursday Island, Australia; ${ }^{2}$ James Cook University, Cairns, Australia; ${ }^{3}$ Cairns Hospital and \\ Hinterland Health Service, Cairns, Australia; ${ }^{4}$ The Kirby Institute, Sydney, Australia
}

\begin{abstract}
Rheumatic heart disease (RHD) is almost entirely preventable, but its incidence in indigenous Australians remains one of the highest in the world. A community-based echocardiogram screening program of 862 Torres Strait Islander children identified 25 (2.9\%) new cases of RHD. Among these 25 children, $5 / 7$ (71\%) prior acute rheumatic fever presentations had not been recognized. There was a history of microbiologically confirmed group A Streptococcus infection in 17/25 (68\%) children with RHD compared with 9/25 (36\%) controls (odds ratio [OR] [95\% Cl]: 3.78 [1.17-12.19], $P=0.03)$. This was more likely to be a skin swab (16/25 [64\%] cases versus 6/25 [24\%] controls) than a throat swab (1/25 [4\%] cases versus 3/25 [12\%] controls) (OR [95\% Cl]: 5.33 [1.51-18.90] $[P=0.01])$, supporting a role for skin infection in RHD pathogenesis. Household crowding and unemployment were common in the cohort, emphasizing the need for prioritizing strategies that address the social determinants of health.
\end{abstract}

Acute rheumatic fever (ARF) results from an abnormal autoimmune response to group A Streptococcus (GAS) infections in genetically susceptible populations. ${ }^{1}$ Episodes of ARF can lead to chronic damage of the heart valves; this is known as rheumatic heart disease (RHD). RHD is a disease of disadvantage and is almost completely preventable. ${ }^{1,2}$ Improvements in the standard of living and treatment of GAS infections mean that RHD is now very rarely diagnosed in high-income countries. However, despite Australia's well-resourced, universal healthcare system, its Indigenous Aboriginal and Torres Strait Islander population continue to bear a disproportionate burden of RHD; the estimated prevalence of the disease in Aboriginal and Torres Strait Islander Australians is 7.9/1,000-one of the highest reported in the world. ${ }^{3}$ Rheumatic heart disease continues to be diagnosed in Indigenous Australians at a rate of 49.9 new cases/100,000 annually. ${ }^{3}$ Between 2013 and 2017, $94 \%$ of new RHD cases in Australia were diagnosed in Indigenous Australians, even though they represent only $3.3 \%$ of the national population. ${ }^{3}$

This continuing epidemic is strongly linked to the socioeconomic disadvantage that many Indigenous Australians still face. There is a growing understanding that although there have been significant advances in the recognition and medical management of RHD, further progress will only be made by addressing the underlying socioeconomic factors that increase the likelihood of GAS infection and that delay access to medical care and appropriate antibiotic therapy.

Although RHD in Aboriginal Australians has been studied extensively, ${ }^{1,4}$ relatively little is known about the epidemiology of RHD in Torres Strait Islander Australians. Aboriginal and Torres Strait Islander Australians face many similar challenges, but the two populations are socioculturally distinct, live in different parts of the country, and have very different histories. ${ }^{5}$ It is therefore important to specifically examine the social, economic, and behavioral factors that might increase their risk of RHD. Only then will healthcare providers-in close consultation with Torres Strait Islander communities-be able

*Address correspondence to Josh Hanson, The Kirby Institute, University of New South Wales, Sydney, Kensington 2052, Australia. E-mail: jhanson@kirby.unsw.edu to develop culturally appropriate strategies to prevent disease and to improve patient outcomes.

This retrospective study aimed to identify the clinical, demographic, and socioeconomic characteristics of Torres Strait Islander children, newly diagnosed with RHD, that might be used to inform strategies to reduce local RHD incidence. The study used data collected during a community screening program performed in school children aged 5-18 years, without known RHD, in the Torres Strait Islands in the Torres Strait Islands in July 2018 (Figure 1). Two pediatric cardiologists and an echocardiographer with experience in the diagnosis of RHD performed echocardiograms after obtaining informed consent from a parent or guardian. Parasternal long and short axis views with apical 4- and 5-chamber views were collected, looking for valvular pathology and congenital heart disease. Children were defined as having definite RHD if they satisfied the WHO criteria for the disease. ${ }^{6}$ All children diagnosed with RHD were matched by age ( \pm 2 years), geographic location, and gender (where possible) with children who had a normal screening echocardiogram. The children's demographics, socioeconomic indices, and details of previous clinic presentations were collected from medical records, where these were available. Acute rheumatic fever was defined using modified Jones criteria described in Australian guidelines. Appropriate antibiotic therapy was also defined according to these guidelines. ${ }^{7}$

Data were entered into an electronic database (Microsoft Excel, Microsoft Corp., Redmond, WA) and were analyzed using statistical software (Stata 14.2, StataCorp, College Station, TX). Groups were compared using the Kruskal-Wallis, chi-squared, or Fisher's exact test, where appropriate. Odds ratios were calculated using logistic regression; conditional logistic regression was used to control for multiple observations in individual patients. The Far North Queensland Human Research and Ethics Committee provided ethical approval for the study (HREC/18/QCH/91). The requirement for informed consent was waived, given the data's retrospective nature and its aggregated presentation. To prevent stigmatization, the names of individual communities were deidentified. 


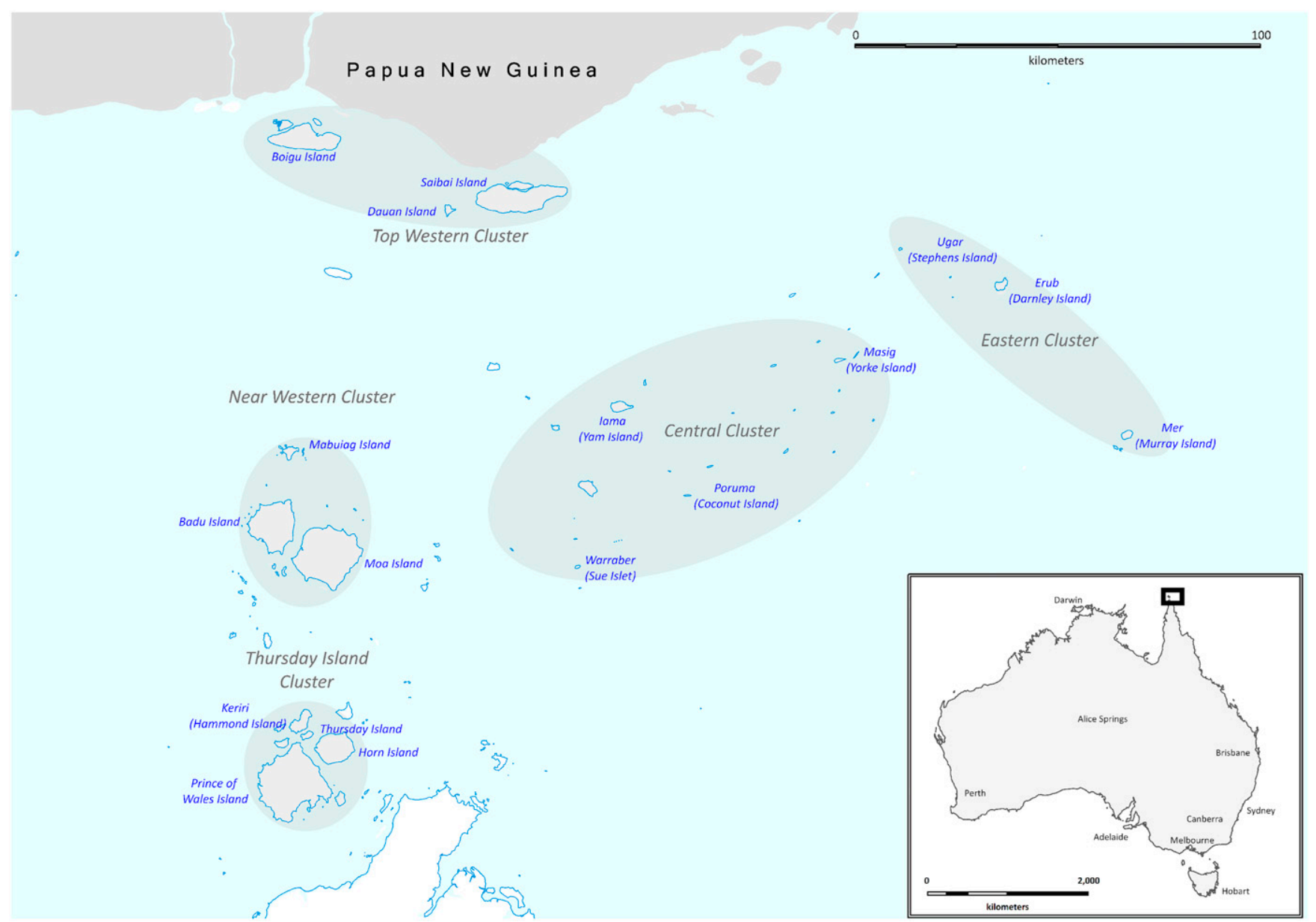

FIgURE 1. Map of Torres Strait Islands, Australia. This figure appears in color at www.ajtmh.org.

There were 862 children screened over 9 days on 12 islands. The children's median (interquartile range [IQR]) age was 10 (8-12) years; 424/862 (49\%) were female, and 837/862 (97\%) identified as Torres Strait Islander Australians. The program identified 25 new cases of RHD, and all were asymptomatic. All cases were among children identifying as Torres Strait Islanders. Cases were spread evenly across all the islands visited by the screening team. There were no cases of severe valvular disease.

The medical records of the 25 patients with RHD were compared with those of 25 age-, gender-, and locationmatched controls (Table 1). Three (12\%) of the 25 with RHD had a prior presentation consistent with ARF compared with $0 /$ 25 controls $(P=0.24)$. One of these three children had their single episode treated appropriately and was referred for specialist review, another had only one of their five presentations treated appropriately, and in the remaining patient, the diagnosis of ARF had been missed. The median (IQR) number of people living in the households of children with RHD was six (4-7) compared with seven $(5-8)$ in the controls $(P=0.24)$. There was no employed adult in the household of the $7 / 23$ (30\%) children with RHD, compared with 10/22 (45\%) controls in whom these data were available $(P=0.37)$.

There was a history of $\geq 1$ healthcare presentation with sore throat in 11/25 (44\%) children subsequently diagnosed with RHD compared with $13 / 25$ (56\%) of the controls $(P=0.57)$. The median (IQR, range) number of sore throat presentations was zero $(0-2,0-6)$ in the children subsequently diagnosed with $\mathrm{RHD}$, compared with one (0-1, 0-9) among controls (OR [95\% $\mathrm{Cl}$ : 0.90 [0.66-1.23], $P=0.51)$. Only eight $(31 \%)$ of the 26 presentations of sore throat in the children subsequently diagnosed with RHD had been treated appropriately compared with 25/35 (71\%) in their controls (OR [95\% Cl]: 0.41 [0.14-1.25], $P=0.11$ ).

There was a history of $\geq 1$ healthcare presentation with skin sores in $18 / 25$ patients (72\%) who were subsequently diagnosed with RHD compared with 16/25 (64\%) of their controls $(P=0.54)$. The median (IQR, range) number of skin sore presentations was two $(0-5,0-13)$ in the children who were subsequently diagnosed with $\mathrm{RHD}$, compared with one (0-4, $0-12$ ) among controls (OR [95\% Cl]: 1.08 [0.92-1.28], $P=$ 0.33). Only $72(81 \%)$ of the 89 presentations with sore throat presentations of sore throat in the children subsequently diagnosed with RHD had been treated appropriately compared with 58/64 (91\%) in their controls (OR [95\% Cl]: 1.07 [0.88-1.30], $P=0.51)$.

Children with RHD were more likely to have a history of a microbiologically confirmed GAS infection 17/25 (68\%) compared with 9/25 (24\%) controls (OR [95\% Cl]: 3.78 [1.17-12.19], $P=0.03)$. However, GAS was identified on skin swab in 22/50 (44\%) compared with only 4/50 (8\%) who had a prior throat swab positive for GAS (OR [95\% Cl]: 5.33 
TABLE 1

Comparison of the demographic and clinical features of children diagnosed with RHD and their age-matched controls

\begin{tabular}{|c|c|c|c|c|}
\hline Variable & Children with RHD $(n=25)$ & Controls $(n=25)$ & Odds ratio $(95 \% \mathrm{Cl})$ & $P$-value \\
\hline Personal history of ARF & $3(12 \%)$ & 0 & - & 0.24 \\
\hline Family history of ARF/RHD & $1(4 \%)$ & $1(4 \%)$ & $1.00(0.06-16.93)$ & 1.0 \\
\hline Number of people resident in household & $6(4-7)$ & $7(5-8)$ & - & 0.24 \\
\hline $\begin{array}{l}\text { No employed adult resident in the } \\
\text { household }\end{array}$ & 7/23 (30\%) & $10 / 22(45 \%)$ & $0.53(0.15-1.78)$ & 0.30 \\
\hline $\begin{array}{l}\geq 1 \text { previous healthcare presentation with } \\
\text { sore throat }\end{array}$ & $11 / 25(44 \%)$ & $13 / 25(52 \%)$ & $0.72(0.24-2.21)$ & 0.57 \\
\hline $\begin{array}{l}\geq 1 \text { previous healthcare presentation with } \\
\text { skin sore }\end{array}$ & $18 / 25(72 \%)$ & $16 / 25(64 \%)$ & $1.45(0.44-4.78)$ & 0.55 \\
\hline $\begin{array}{l}\text { Median (IQR) number of presentations } \\
\text { with sore throat }\end{array}$ & $0(0-2)$ & $1(0-1)$ & - & 0.73 \\
\hline $\begin{array}{l}\text { Median (IQR) number of presentations } \\
\text { with skin sore }\end{array}$ & $2(0-5)$ & $1(0-4)$ & - & 0.38 \\
\hline $\begin{array}{l}\text { Number of presentations with sore throat } \\
\text { given appropriate antibiotics }\end{array}$ & $8 / 26(31 \%)$ & $25 / 35(71 \%)$ & $0.50(0.13-2.0)$ & 0.33 \\
\hline $\begin{array}{l}\text { Number of presentations with skin sores } \\
\text { given appropriate antibiotics }\end{array}$ & $72 / 89(81 \%)$ & $58 / 64(91 \%)$ & $0.67(0.19-2.36)$ & 0.53 \\
\hline GAS isolated on throat swab & $1(4 \%)$ & $3(12 \%)$ & $0.31(0.03-3.16)$ & 0.32 \\
\hline GAS isolated on skin swab & $16(64 \%)$ & $6(24 \%)$ & $5.62(1.65-19.23)$ & 0.006 \\
\hline $\begin{array}{l}\text { GAS isolated on either skin or throat } \\
\text { cultures }\end{array}$ & $17(68 \%)$ & $9(36 \%)$ & $3.78(1.17-12.19)$ & 0.03 \\
\hline
\end{tabular}

[1.50-18.9], $P=0.01)$. The 25 new cases of definite RHD-when added to the 21 existing local RHD casestranslated into a prevalence of 34 confirmed RHD cases per 1,000 population among those aged between 5 and 20 years in the region. ${ }^{8}$

This is the first dedicated study to examine, in detail, the burden of RHD in a Torres Strait Islander population. Screening identified new cases of RHD in $2.9 \%$ of the screened Torres Strait Islander children, a rate that is comparable with the prevalence seen in Aboriginal Australian, Maori, and Timor-Leste children. ${ }^{9-11}$

It is concerning that despite ongoing local education about ARF, symptomatic patients are not presenting for care, and even when they do, the condition is still frequently not being recognized. In 2007, it was estimated that over $20 \%$ of ARF cases in the region were being missed, whereas in this series, only two of seven prior healthcare presentations with ARF were recognized. ${ }^{12}$ The study also identified missed opportunities for disease prevention: although Australian RHD guidelines recommend that children at high risk of ARF with sore throat should be treated empirically with antibiotics, ${ }^{4}$ almost half the children's presentations with sore throat did not receive this treatment. This is likely to be partly explained by the concerted effort to reduce the prescription of antibiotics for upper respiratory tract infections in Australia and by clinicians-working on short-term contracts in the Torres Strait Islands-who may be unfamiliar with recommendations for high-risk populations. ${ }^{13,14}$ It is also likely to be explained by the fact that in this remote region of Australia, many island communities are visited by a doctor only fortnightly. The role of clinical scoring systems and rapid diagnostic tests in identifying GAS tonsillitis requires evaluation, but until their utility is established, these data provide more support for prompt empirical antibiotic therapy by local healthcare workers. ${ }^{4}$

It has been hypothesized that GAS skin infections may play a role in the pathogenesis of RHD in Australian Aboriginal and Pacific Island populations, although this link is yet to be confirmed. ${ }^{15}$ Previous studies have illustrated high rates of skin infections and low rates of symptomatic pharyngitis in remote Aboriginal Australian communities where RHD is highly prevalent. ${ }^{16}$ It was therefore notable that presentations with skin sores were more common than presentations with sore throat in this cohort and that children with RHD were more likely to have GAS cultured from a skin swab than a throat swab.

However, while the clinical management of these children was suboptimal, purely medical approaches are unlikely to significantly reduce the local burden of RHD. Primordial prevention-which addresses the social determinants of health-is likely to be far more cost-effective and would also be expected to reduce the burden of other diseases of disadvantage seen commonly in Torres Strait Islander children, including Staphylococcus aureus infection and suppurative otitis media. ${ }^{2,17-19}$ In this small study, it was not possible to examine all the socioeconomic, environmental, and behavioral factors that influence GAS incidence; however, significant crowding and very high rates of unemployment were reported. There was no employed adult in the homes of almost $40 \%$ of the participants, which compares with the national unemployment figure of $5.4 \%$ at the time of the screening program. ${ }^{20}$ Meanwhile, there were an average number of 6.9 people per household in this study compared with a national average of 2.6. ${ }^{8}$ The Australian Bureau of Statistics has developed a measure of socioeconomic disadvantage, the Socio-Economic Indexes for Areas (SEIFA) score, and all the islands of the Torres Strait have a score in the lowest quintile in the country. ${ }^{21}$ The SEIFA score has been shown to have a strong inverse correlation with the prevalence of RHD in the region. ${ }^{22}$

This study has many limitations. The small sample size increases the risk of type 2 statistical errors, and its retrospective design increases the likelihood of incomplete or inaccurate data. There are 2,327 individuals aged 5-19 years living on the 17 inhabited Torres Strait Islands, and the screening program was not able to reach them all. ${ }^{8}$ The difficulty in accessing all these children underscores the challenges of providing health care to such a geographically dispersed population. 
However, this study demonstrates that new cases of RHD are still being identified in Torres Strait Islander children, that ARF remains under-recognized, and that opportunities for primary prevention are being missed. The study underscores the value of echocardiographic screening programs in defining RHD burden and by highlighting nonadherence to published guidelines-in particular, the lower threshold for antibiotic treatment of sore throat in high-risk populationsthe study offers potential strategies that might reduce ARF and RHD incidence. However, for effective and substantial progress to be made, community-led strategies are crucial if we are to address the socioeconomic and environmental factors that are known to increase the risk of GAS infection and the many other diseases that disproportionately affect Torres Strait Islander Australians.

Received July 13, 2020. Accepted for publication November 25, 2020.

Published online January 11, 2021.

Acknowledgment: We thank Peter Horne (Cairns Tropical Public Health Unit) for his assistance with the production of Figure 1.

Authors' addresses: Allison Hempenstall, Amylou Browne, Ella Kris, Hylda Wapau, and Pelista Pilot, Torres and Cape Hospital and Health Service, Thursday Island, Australia, E-mails: allison.hempenstall@health.qld.gov.au, amylou.browne@health.qld.gov.au, ella.kris@tsirc.qld.gov.au, hylda.wapau@ health.qld.gov.au, and pilotpel06@gmail.com. Erin Howell, Katherine Kang, Ken W. T. Chau, Simon Smith, and Benjamin Reeves, Cairns Hospital and Hinterland Health Service, Cairns, Australia, E-mails: erinhowell21@ gmail.com, katherine.kang@health.qld.gov.au, ken.chau@health.qld.gov.au, simon.smith2@health.qld.gov.au, and benjamin.reeves@health.qld.gov.au. Josh Hanson, The Kirby Institute, University of New South Wales, Kensington, Sydney, Australia, E-mail: jhanson@kirby.unsw.edu.

\section{REFERENCES}

1. Carapetis JR et al., 2016. Acute rheumatic fever and rheumatic heart disease. Nat Rev Dis Primers 2: 15084.

2. Coffey PM, Ralph AP, Krause VL, 2018. The role of social determinants of health in the risk and prevention of group A streptococcal infection, acute rheumatic fever and rheumatic heart disease: a systematic review. PLoS Negl Trop Dis 12: e0006577.

3. Australian Health Ministers' Advisory Council, 2017. Aboriginal and Torres Strait Islander Health Performance Framework 2017 Report. Canberra, Australia: Australian Health Ministers' Advisory Council.

4. Ralph AP, Noonan S, Wade V, Currie BJ, 2020. The 2020 Australian guideline for prevention, diagnosis and management of acute rheumatic fever and rheumatic heart disease. Med J Aust 2020 Nov 15. doi: 0.5694/mja2.50851.

5. Torres Strait Islander Regional Council, 2016. Culture, Protocols and Heritage. Available at: www.tsirc.qld.gov.au/ourcommunities/culture-protocols-heritage. Accessed June 1, 2020.

6. Remenyi B et al., 2012. World Heart Federation criteria for echocardiographic diagnosis of rheumatic heart disease--an evidence-based guideline. Nat Rev Cardiol 9: 297-309.

7. Carapetis J et al., 2012. The Australian Guideline for Prevention, Diagnosis and Management of Acute Rheumatic Fever and
Rheumatic Heart Disease, 2nd edition. Darwin, Australia: RHD Australia. Available at: https://researchers.cdu.edu.au/en/ publications/the-australian-guideline-for-prevention-diagnosisand-management.

8. Australian Bureau of Statistics, 2016.2016 Census. Available at: https:// quickstats.censusdata.abs.gov.au/census_services/getproduct/ census/2016/quickstat/POA4875. Accessed November 11, 2020.

9. Davis $\mathrm{K}$ et al., 2018. Rheumatic heart disease in Timor-Leste school students: an echocardiography-based prevalence study. Med J Aust 208: 303-307.

10. White H, Walsh W, Brown A, Riddell T, Tonkin A, Jeremy R, Brieger D, Zeitz C, Kritharides L, 2010. Rheumatic heart disease in indigenous populations. Heart Lung Circ 19: 273-281.

11. Francis JR et al., 2020. Hyperendemic rheumatic heart disease in a remote Australian town identified by echocardiographic screening. Med J Aust 213: 118-123.

12. -, 2007. Why are medical practitioners in north Queensland missing cases of acute rheumatic fever? Trop Popul Health Unit Netw Commun Dis Control News/ 55: 1-4.

13. Wu J, Taylor D, Ovchinikova L, Heaney A, Morgan T, Dartnell J, Holbrook R, Humphreys L, Weekes L, Blogg S, 2018. Relationship between antimicrobial-resistance programs and antibiotic dispensing for upper respiratory tract infection: an analysis of Australian data between 2004 and 2015. J Int Med Res 46: 1326-1338.

14. Tarca AJ, Hand RM, Wyber R, 2019. Call for a national sore throat guideline. Med J Aust 210: 477-477.e1.

15. Parks T, Smeesters PR, Steer AC, 2012. Streptococcal skin infection and rheumatic heart disease. Curr Opin Infect Dis 25: 145-153.

16. McDonald MI, Towers RJ, Andrews RM, Benger N, Currie BJ, Carapetis JR, 2006. Low rates of streptococcal pharyngitis and high rates of pyoderma in Australian aboriginal communities where acute rheumatic fever is hyperendemic. Clin Infect Dis 43: 683-689.

17. Guthridge I, Smith S, Horne P, Hanson J, 2019. Increasing prevalence of methicillin-resistant Staphylococcus aureus in remote Australian communities: implications for patients and clinicians. Pathology 51: 428-431.

18. Nofz L, Koppen J, De Alwis N, Smith S, Hanson J, 2019. The microbiology of ear cultures in a high-burden setting in tropical Australia: implications for clinicians. Clin Otolaryngol 44: 1195-1200.

19. Smith S, Russell D, Horne $P$, Hanson J, 2019. HTLV-1 is rare in Far North Queensland despite a significant burden of classically associated diseases. Pathology 51: 91-94.

20. Australian Bureau of Statistics, 2018. Labour Force, Australia. Available at: https://www.abs.gov.au/ausstats/abs@ .nsf/lookup/6202.0Media\%20Release1Jun\%202018. Accessed November 24, 2020.

21. Australian Bureau of Statistics, 2016. Census of Population and Housing: Socio-Economic Indexes for Areas, Australia. Available at: https://www.abs.gov.au/ausstats/abs@.nsf/Lookup/ by\%20Subject/2033.0.55.001 2016 Main\%20Features What\% 20is\%20SEIFA\%3f 8. Accessed June 1, 2020.

22. Kang K, Chau K, Howell E, Anderson E, Smith S, Davis T, Starmer G, Hanson J, 2020. The temporospatial epidemiology of rheumatic heart disease in Far North Queensland, tropical Australia 1997-2017; impact of socioeconomic status on disease burden, severity, and access to care. PLoS Negl Trop Dis, In press. 